\title{
The Effects of Mobile Service Quality and Technology Compatibility on Users' Perceived Playfulness
}

\author{
Felix B. Tan ${ }^{1}$ and Jacky P.C. Chou ${ }^{1}$ \\ ${ }^{1}$ School of Computing and Mathematical Sciences \\ Private Bag 92006, Auckland 1020, New Zealand \\ felix.tan@aut.ac.nz,ggyy@xtra.co.nz
}

\begin{abstract}
There is a growing body of research into Mobile Information and Entertainment Services in recent years. This study explored the effect of mobile service quality and its compatibility to mobile technology on users' perceived playfulness toward these services. Perceived Service Quality and Perceived Technology Compatibility were found to influence users' Perceived Playfulness. Furthermore, Perceived Technology Compatibility significantly mediated the relationship between Perceived Service Quality and Perceived Playfulness. This suggests that service attributes need to be aligned with mobile phone functionalities to enhance user's Perceived Playfulness. In addition, Perceived Usefulness, Ease of Use and Personalization were the three most important service quality aspects that influenced Perceived Playfulness.
\end{abstract}

Keywords: Perceived Service Quality, Perceived Technology Compatibility, Perceived Playfulness, Mobile Information and Entertainment Services.

\section{Introduction}

The introduction of real-time video-calling, Mobile TV, Multi Media Services and many other exciting offerings has seen the realization of faster mobile internet services through Third Generation Mobile Networking Technologies (3G). Among these services, mobile information and entertainment services (MIES) are gaining in popularity among mobile phone users [1]. With increasing popularity, companies providing MIES will therefore benefit from sound conceptual and empirical research that enhance the understanding of the factors critical to user acceptance of MIES. MIES can be defined as "the delivery of information and entertainment from specially formatted content sources (Internet sites, SMS, MMS, etc.) via the mobile telecommunication network to a mobile user".

Recent research has shown better service quality will lead to better acceptance of these services by users [2]. Methlie and Pedersen [3] suggested that there are two kinds of service attributes: intrinsic attributes and extrinsic attributes. While extrinsic attributes emerge from the networks that provide and use network services, intrinsic attributes refer to the inherent attributes of the service itself. This study focused on the intrinsic service attributes and treated Perceived Service Quality as a multidimensional construct that integrated these intrinsic service attributes. 
Research into mobile internet to date has by and large focused on extrinsic use of these services (e.g. productivity and usefulness) and has largely ignored important intrinsic uses. To better understand users' acceptance of MIES, we argue that it is equally important to examine an intrinsic motivator "Perceived Playfulness". Several studies have demonstrated the importance of this construct especially in the wider internet context [4] as well as mobile internet services [5, 6]. This construct was proposed by Moon and Kim [7] based on the theory of flow. Flow has been an important theory when studying user intrinsic use of technology.

Mobile technology is the enabling force for the development of new mobile internet services. In the field of mobile commerce, mobile technologies have been a major research topic $[1,8]$. Lu et al. [8] pointed out that the main problem can be attributed to the incompatibility between mobile devices and mobile data services.

In this study, we proposed Perceived Service Quality and Perceived Technology Compatibility as the two antecedents of Perceived Playfulness in the context of MIES. Perceived Service Quality was treated as a higher order factor formed by seven other intrinsic factors: Perceived Usefulness, Perceived Ease of Use, Content, Variety, Feedback, Experimentation and Personalization. The effect of the compatibility between service attributes and mobile phone functionalities on Perceived Playfulness was also tested.

\section{Theoretical Perspective}

\subsection{Perceived Playfulness}

According to Moon and Kim [7], Perceived Playfulness can be defined as: "The extent to which the individual perceives that his or her attention is focused on the interaction with the World-Wide-Web; is curious during the interaction; and finds the interaction intrinsically enjoyable or interesting".

Based on the theory of Flow, they considered playfulness as an intrinsic belief or motive, which is shaped by the individual's experience with the environment. Flow represents an optimal experience when a person is unconsciously engaged in an activity such that $\mathrm{s} /$ he becomes so engrossed thereby losing the sense of self. Flow experience can usually occur in structured activities such as games, ritual events, sports, artistic performances and etc [9].

Several researchers have studied perceived playfulness in the context of mobile internet services. In their study of four mobile services, Nysveen et al. [5] found enjoyment plays an important role in determining user acceptance of these services. Fang et al. [6] categorized mobile tasks that can be performed on handheld devices into three broad types: general task, transactional task and gaming task. They found Perceived Playfulness was significant when users perform gaming tasks. These results suggest Perceived Playfulness played a distinct role in user's favourable reception of mobile internet services. 


\subsection{Perceived Service Quality}

Perceived Service Quality (PSQ) has been recognized as a major driver of satisfaction in mobile entertainment services [2]. In this study, Perceived Service Quality is proposed as a multidimensional second order construct that integrates intrinsic service attributes that were identified in literature on intrinsic motivators and mobile commerce. This construct assembles the core values that encourage users to develop positive image toward MIES and most likely lead to the adoption of these services. Table 1 presents these first order factors.

Table 1. Multidimensionality of Perceived Service Quality

\begin{tabular}{|l|l|}
\hline Dimensions of PSQ & Found to be important to \\
\hline Perceived Usefulness & $\begin{array}{l}\text { Perceived Playfulness [4]; Enjoyment [10]; Cognitive Absorption } \\
{[11]}\end{array}$ \\
\hline Perceived Ease of Use & $\begin{array}{l}\text { Experiencing Flow [12]; Motivation to use Internet [13]; Gaming } \\
\text { Tasks [6] }\end{array}$ \\
\hline Content & $\begin{array}{l}\text { Wireless and Web Context [14]; Mobile Information Quality [15]; } \\
\text { Overall Superiority of Services [2] }\end{array}$ \\
\hline Variety & $\begin{array}{l}\text { Audience Engagement [16]; Furthering the State of Flow [17]; } \\
\text { Perceived Value of Mobile Services [3] }\end{array}$ \\
\hline Feedback & $\begin{array}{l}\text { A Computer-mediated Environment [16, 18]; Building Links with } \\
\text { Customers [17] }\end{array}$ \\
\hline Experimentation & Encouraging Exploratory Behaviors Leading to Flow [4, 19] \\
\hline Personalization & Services Quality [20] \\
\hline
\end{tabular}

In terms of the nature of PSQ as a second order unitary construct, the relationships among the first-order factors should be mutually reinforcing [21]. These factors cumulatively contribute to a unitary higher-order structure, which can more parsimoniously explain a complex construct. Given that users are likely to place different weights on the seven factors, the importance assigned to each factor is proposed to influence PSQ in a formative fashion. According to Chin [22], a change in any first order factor does not necessarily imply a similar directional change in others for a formative model.

\subsection{Perceived Technology Compatibility}

A new construct called Perceived Technology Compatibility (PTC) is proposed based on Lu et al's [23] work. PTC is defined as "the perceived degree of integration between mobile information and entertainment services and mobile phones". Given that enhancing the functionalities of mobile phones is a means of satisfying user requirements, it is more appropriate to look at the compatibility between MIES and mobile phones.

The impact of system characteristics has been widely recognized in studies on technology user acceptance $[24,25]$. Studies on the effects of system characteristics on intrinsic motivators can be traced back to Trevino and Webster [12]. A positive influence of perceived technology characteristics on flow was found in their study. 
Webster et al. [19] suggested users' perceptions of the program's flexibility and modifiability may contribute to flow experience. The result indicated that if the functionalities of a technology were more aligned with users' needs it would facilitate their experiences of flow. Similarly, Agarwal and Karahanna [11] reported that technologies perceived to be compatible with various aspects of individual's experiences and work styles were likely to induce feelings of familiarity and positive affect.

\subsection{The Mediating Effect of Perceived Technology Compatibility}

Improving user satisfaction is the ultimate goal of improving application performance on wireless devices [8]. Lin et al. [26] incorporated Perceived Playfulness in to the expectation-confirmation theory and found the confirmation of expectation and perceived performance would lead to Perceived Playfulness which in turn drove user's satisfaction. However, perceived hardware and software functionalities have been identified as one of the main perceived obstacles by mobile users $[10,27]$. According to Levy [28], the overall performance requirements have raised users' expectations about digital entertainment services, which included mobile phones. Ironically, users expecting more from their phones were influenced by the extensive marketing campaigns of service providers. Koivisto and Urbaczewski [29] found inconsistent quality of services perceived and delivered caused stress and discomfort when users interacted with mobile internet services.

Most research conducted on mobile internet elaborated on technology requirements without discussing important end-user needs. Kettinger and Lee [30] presented a model where gaps can occur between IT services delivered and user's perception of the service expected. However, their study was focused on building a strategic model rather than on IT-User relationship. In his study of human computer interaction, Te'eni [31] pointed out perceived complexities always existed as an intermediate variable between users' perception of the system and system itself. Two variables, mode of presentation and number of windows, were examined in his study. His findings suggested the congruence between user's perception and the functionalities offered would subsequently impact on outcome. Their study further pointed out that the usefulness of information/system characteristics perceived by users is a major component in the formation of perceived complexities. Mobile phones serve as the entry point for users to interact with MIES. If their perceived compatibility between MIES and mobile phone is consistent with their expectation, it will reinforce a positive image of MIES. Thus, the intermediate role of Perceived Technology Compatibility stimulated our interest to test its mediating effect between Perceived Service Quality and Perceived Playfulness in the MIES context. If the mediating effect is significant, the influence of Perceived Service Quality on Perceived Playfulness will be lessened significantly in the presence of Perceived Technology Compatibility. 


\section{Development of the Research Model and Hypotheses}

From the discussion above, the research model in Fig 1 is proposed. Note that Perceived Service Quality is proposed as a second order formative factor (H1). Both Perceived Service Quality (H2) and Perceived Technology Compatibility (H3a and $\mathrm{H} 3 \mathrm{~b}$ ) are proposed to exert significant influences over Perceived Playfulness. However, the latter also serves to be a mediating factor which mediates the effect of Perceived Service Quality on Perceived Playfulness (H3c).

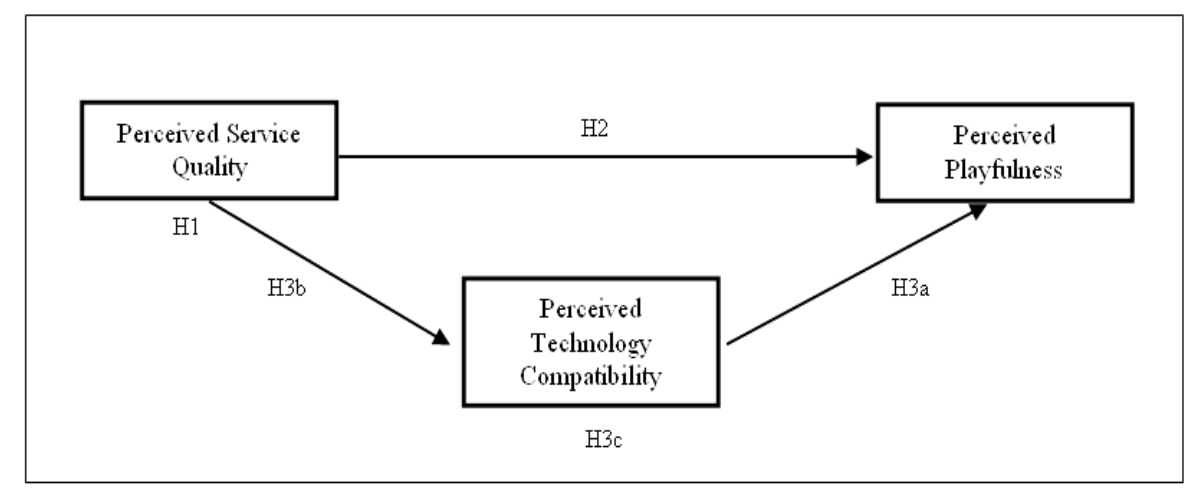

Fig. 1. Research Model

\section{Research Design}

The sample consisted of university students who attend information system and business lectures at a university. Students from six classes were invited to do the questionnaires. A total of 186 questionnaires were returned. Incomplete questionnaires were discarded, leaving 149 usable samples. The participants were 99 males and 55 females. In terms of phone categories, about $60 \%$ of all the respondents specified they have WAP-enabled mobile phone or GPRS phone. Respondents holding a $3 \mathrm{G}$ mobile phone came second in the list, with a significant proportion of $12 \%$ among the respondents. Users of the CDMA mobile phone are relatively few, only $7 \%$ compared to other phone categories.

In terms of measurement, all items were constructed as agree-disagree statements on a seven-point Likert scale. A small pilot study was conducted and some items revised on the basis of pilot. The measures were adapted from various sources in the literature $[4,7,14,16,19,23,32]$. 


\section{Data Analysis and Results}

The data analysis technique employed in this study is Partial Least Squares (PLS). The PLS approach provides a means for directly estimating latent variable component scores as well as estimate formative constructs using component-based algorithms [22].

\subsection{Measurement Validation}

To measure internal consistency of a given block of indicators, internal composite reliability (ICR) scores were obtained through PLS-GRAPH to assess the reliabilities of each latent variable. All latent variables in our model have internal consistencies greater than 0.7 which indicates all constructs have high reliabilities [33]. At the same time, the average variance extracted (AVE) of each latent variable in our model is more than 0.50 , which is deemed satisfactory [33]. All measures exhibited high convergent validity with t-values of loadings above 1.96 [34] and high discriminant validities through bootstrap re-sampling [33].

Further, the results obtained show all PSQ indicators have a coefficient significantly different from zero $(\mathrm{p}<0.01)$, with a minimum t-value of 5.0293 [35]. It has also been noticed that the first two measures and the last measure of PTC (PTC1, PTC2 and PTC 10) and the second measure of PP (PP2) exhibit very low loadings. As a result, PTC1, PTC2 and PTC 10 and PP2 were eliminated in the revised model. The test of PSQ was carried out with the same set of tests in Chin [22] and Pavlou and El Sawy [21]. Since PSQ is formative in nature, each first order factor represents an aspect of PSQ. Therefore, low correlations among first order factors are expected [36]. The correlations between those first order factors and PSQ were tested. The results indicate all first order factors are highly correlated with PSQ $(p<0.01)$.

\subsection{The Structural Model}

The direct influence of PSQ has a significant impact on PP $(\beta=0.719 ; \mathrm{t}=15.7557)$, as shown in Fig 2(a). Similarly, in Fig 2(b), PTC also significantly influences PP. To validate the key mediating role of PTC, three competing models were compared. The mediation test strictly followed the guidelines provided in Baron and Kenny [37]. In Fig 2 (a), a strong and positive impact of PSQ on PP was shown. In terms of variance explained, PSQ predicts $51.3 \%$ of variances in $\mathrm{PP}\left(\mathrm{R}^{2}=0.513\right)$. Theses provide support for Hypothesis 1 and 2.

Next, PTC was introduced as a mediating factor between PSQ and PP. As Fig 2(c) attests, the second model mediated by PTC is superior to the first model in terms of variance explained. The model with both PTC and PSQ has a $\mathrm{R}^{2}$ value of 0.539 which is better than the first two models illustrating this model provides a richer picture for describing users' PP. Also, although the effect of PSQ on PP was still significant, the strength of the effect was reduced (from $\beta=0.719 ; \mathrm{t}=15.7557$ to $\beta=0.562 ; \mathrm{t}=5.4401$ ), thus $\mathrm{H} 2 \mathrm{c}$ is partially supported. This outcome was expected because its influence on PP is mediated by PTC. Furthermore, the significant impact of PSQ on PTC and PP 
also provides evidence of external validities for PSQ as a formative second order construct [35].

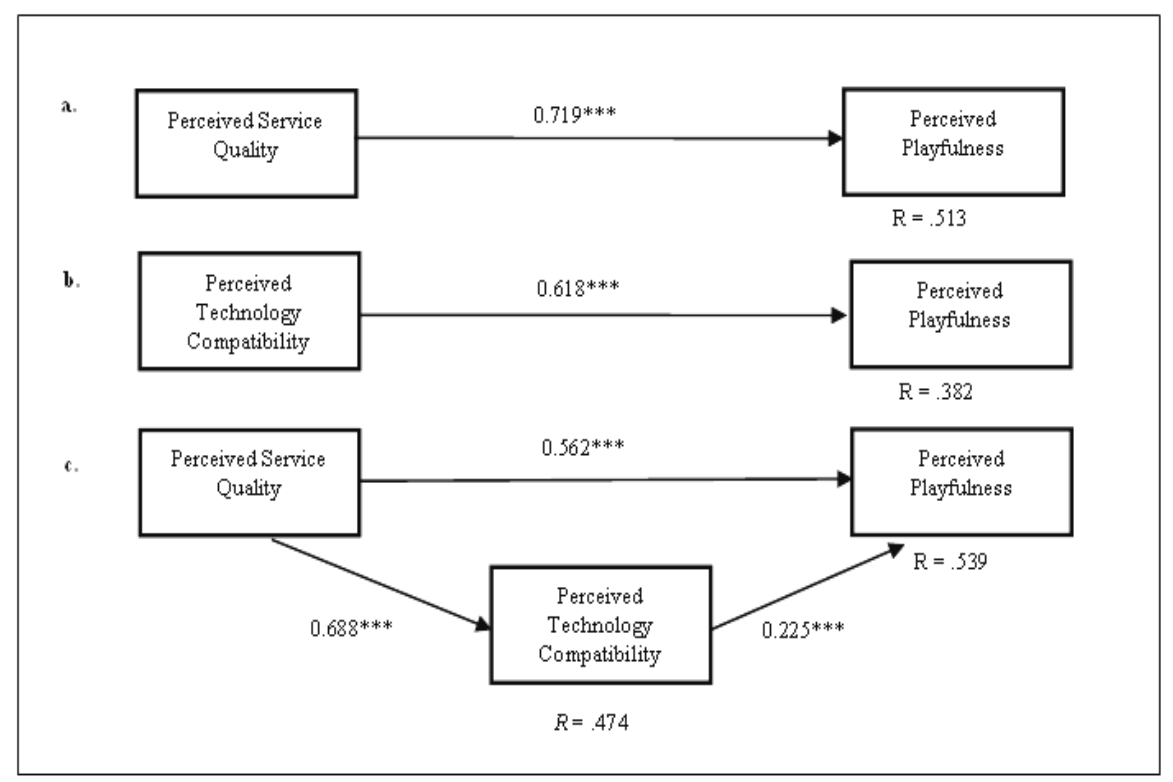

Fig. 2. Mediating Role of Perceived Technology Compatibility

\section{Discussion and Conclusion}

In this study, PSQ as a higher order factor has been validated in this studyanswering Hypothesis 1. It also significantly impacts on PP. Thus Hypothesis 2 is supported. To probe deeper into the nature of PSQ, this study tested its underlying factors. Our findings suggest Perceived Usefulness, Ease of Use and Personalization with relatively higher path magnitudes are the major determinants of PSQ. To create a positive image of MIES for mobile phone users, service providers should focus on improving the usefulness and ease of use of current MIES service offerings. More importantly, they should explore the opportunities to offer more personalized services. Current practice among service providers seems to be offering one for all standard wireless portals for MIES (e.g. Vodafone Live!). Other dimensions of PSQ such as Content, Variety, and Experimentation are all significantly related to Perceived Service Quality. However, they do not weigh as importantly as Perceived Usefulness, Ease of Use and Personalization. This study empirically tested all these factors and provides evidence of the existence of this latent construct. It indicates the user forms an overall perception of service quality toward MIES rather than an attitude based on a single service attribute. 
It is noteworthy that PTC mediates the effect of PSQ on PP. The indirect effect of PSQ on PP via PTC provides empirical support for this. Therefore, hypotheses 3a, 3b were supported, whereas $3 \mathrm{c}$ is partially supported. The implication of this is that although users may have higher PSQ of MIES and may experience higher PP, this has less significant effect on PP than the effects mediated by PTC. This suggests service providers should emphasize provision of seamless MIES experience through closely integrated mobile phone functionalities and services. While many researchers investigated the direct impacts of service and technology factors in parallel on intention to use mobile internet services $[1,3,10]$, this study provides empirical evidence that PTC mediates the impacts of PSQ on PP.

The most apparent limitation of this study is its cross-sectional nature and the use of a convenience sample. Thus, caution must be exercised when generalizing the findings. This study also focused on the perception of mobile information and entertainment services, but did not examine the adoption of these services. Therefore, the significance of PP in the context of MIES adoption was not examined. Future research on this can shed new light on user adoption of MIES.

In terms of services, Perceived Usefulness, Ease of Use and Personalization are the three most important service quality aspects that influence Perceived Playfulness. Therefore, service providers need to provide useful, user-friendly, and personalized MIES for users. Current practice among service providers seems to be providing a "one site for all" wireless portal. Smyth and Cotter [38] pointed out the need for Personalization to overcome this problem. On the other hand, the existence of PSQ as a second order construct indicates users form overall service quality perceptions rather than judging on the basis of any single service aspect. One of this study's primary contributions is the mediating role of PTC. In the presence of this construct, the significance of PSQ on PP dropped considerably. As Fang et al. [6] attested, service providers need to consider the effect of different tasks and user needs in interface design. Many researchers investigated the direct impacts of service and technological attributes on user's perception and overlooked the interaction between these two factors. This research suggests it is important to pay closer attention to this area.

\section{References}

1. Van de Kar, E. A. M., Maitland, C., Wehn de Montalvo, U., Bouwman, H.: Design guidelines for Mobile Information and Entertainment Services: Based on the Radio538 Ringtunes i-mode Service Case Study. In Sadeh, Norman (Eds.) Proceedings of the Fifth International Conference on Electronic Commerce (2003) 414-422

2. Vlachos, P., Vrehopoulos, A.: Special issue on Consumers' Behavioral Intentions in the Mobile Internet Services Market. The Hermes Newsletter, ELTRUN. Athens University of Economics and Business, available at: http://www.eltrun.aueb.gr (2005)

3. Methlie, L.B., Pedersen, P. E.: Network Effects and Intrinsic Attributes of Mobile Services: Mapping Perceived Service Values on Business Model Choices. Presented at the Hong Kong Mobility Roundtable (2005)

4. Chung, J., Tan, F. B.: Antecedents of Perceived Playfulness: An Exploratory Study on User Acceptance of General Information-Searching Websites. Information \& Management, 41(7) (2004) 869-881 
5. Nysveen, H., Pedersen, P. E., Thorbjørnsen, H.: Intentions to Use Mobile Services: Antecedents and Cross-Service Comparisons. Journal of the Academy of Marketing Science, 33(3) (2005) 330-346

6. Fang, X. W., Chan, S., Brzezinski, J., Xu, S.: Moderating Effects of Task Types on Wireless Technology Acceptance. Journal of Management Information Systems, 22(3) (2006) 123157

7. Moon, J. W., Kim, Y. G.: Extending the TAM for a World-Wide-Web context. Information \& Management, (38)4 (2001) 217-230

8. Raisinghani, V. T., Iyer, S.: Cross-layer Design Optimizations in Wireless Protocol Stacks. Computer Communications, 27 (2004) 720-724

9. Csikszentmihalyi, M.: The Future of Flow. In Csikszentmihalyi, M., \& Csikszentmihalyi, I. S. (Eds.) Optimal Experience: Psychological Studies of Flow in Consciousness. New York (1988) 364-383

10. Pagani, M.: Determinants of Adoption of Third Generation Mobile Multimedia Services. Journal of Interactive Marketing, 18(3) (2004) 46-59

11. Agarwal, R., Karahanna, E.: On the Multi-dimensional Nature of Compatibility Beliefs in Technology Acceptance. Proceedings of the 19th Annual International Conference on Information Systems (1998) 13-16

12. Trevino, L. K., Webster, J.: Flow in Computer-Mediated Communication. Communication Research, 19(5) (1992) 539-573

13. Bruner, G. C., Kumar, A.: Explaining Consumer Acceptance of Handheld Internet Devices, Journal of Business Research, 58 (2005) 553-558

14. Chae, M., Kim, J., Kim, H., Ryu, H.: Information Quality for Mobile Internet Services: A Theoretical Model with Empirical Validation. Electronic Markets, 12(1) (2002) 38-46

15. Vlachos, P., Vrechopoulos, A., Doukidis, G.: Exploring Consumer Attitudes towards Mobile Music Services. International Journal on Media Management, 5(2) (2003) 138-148

16. Webster, J., Ho, H.: Audience Engagement in Multimedia Presentations. The DATA BASE for Advances in Information Systems, 28(2), (1997) 63-77

17. Lee, Y. E., Benbasat, I.: A Framework for the Study of Customer Interface Design Form Mobile Commerce. International Journal of Electronic Commerce, 8(3) (2004) 79-102

18. Hoffman, D. L., Novak T. P.: Marketing in Hypermedia Computer-Mediated Environments: Conceptual Foundations. Journal of Marketing, 60 (1996) 50-68

19. Webster, J., Trevino, L. K., Ryan, L.: The Dimensionality and Correlates of Flow in Human-computer Interactions. Computers in Human Behavior, 9(2) (1993) 411-426

20. Mittal, B., Lassar, W. M.: The Role of Personalization in Service Encounters. Journal of Retailing, 72(1) (1996) 95-109

21. Pavlou, P. A., El Sawy, O. A.: From IT Competence to Competitive Advantage in Turbulent Environments: A Dynamic Capabilities Model. Information Systems Research, (under 3rd round of review). Forthcoming (2005)

22. Chin, W. W.: Issues and Opinion on Structural Equation Modeling. MIS Quarterly, 22(1), (1998) vii-xvi

23. Lu, J., Liu, C., Yu, C. S., Yao, J. E.: Exploring the Factors Associated with Wireless Internet via Mobile Technology Acceptance in Mainland China. Communications of the International Information Management Association, 3(1) (2003) 101-120

24. Davis, F.: User Acceptance of Information Technology: System Characteristics, User Perceptions and Behavioural Impacts. International Journal of Man-Machine Studies, 38 (1993) 475-487

25. Davis, F., Bagozzi, R., Warshaw, P.: User Acceptance of Computer Technology: A Comparison of Two Theoretical Models. Management Science, 35 (1989) 982-1003

26. Lin, C., Wu, S., Tsai, R.: Integrating Perceived Playfulness into Expectation-confirmation Model for Web Portal Context. Information \& Management, 42(5) (2005) 683-693 
27. Lu, J., Yu, C. S., Liu, C., Yao, J. E.: Technology acceptance model for wireless internet. Internet Research: Electronic Networking Applications and Policy, 13(3) (2003) 206-222

28. Levy, M.: Evaluating Digital Entertainment System Performance, IEEE Computer Society Press, 38(7) (2005) 68-72

29. Koivisto, M., Urbaczewski, A.: The Relationship between Quality of Service Perceived and Delivered in Mobile Internet Communications. Information Systems and e-Business Management, 2 (2004) 309-323

30. Kettinger, W., Lee, C.: Exploring a Gap Model of Information Services Quality. Information Resources Management Journal, 8(3) (1995) 5-16

31. Te'eni, D.: Determinants and Consequences of Perceived Complexity in Human-Computer Interaction. Decision Sciences, 20 (1989) 166-181

32. Koufaris, M.: Applying the Technology Acceptance Model and Flow Theory to Online Consumer Behavior. Information Systems Research, 13(2) (2002) 205-23

33. Fornell, C., Larcker, D. F.: Evaluating Structural Equation Models with Unobservable Variables and Measurement Error. Journal of Marketing Research, 18 (1981) 39-50

34. Gefen, D., Straub, D.: A Practical Guide to Factorial Validity Using PLS-Graph: Tutorial and Annotated Example. Communications of the Association for Information Systems, 16(5) (2005) 91-109

35. Diamantopoulos, A., Winklhofer, H.: Index Construction with Formative Indicators: An Alternative to Scale Development. Journal of Marketing Research, 38 (2) (2001) 269-277

36. Gefen, D., Straub, D., Boudreau, M.C.: Structural Equation Modeling and Regression: Guidelines for Research Practice. Communications of the Association for Information Systems, 4(7) (2000) 1-80

37. Baron, R. M., Kenny, D. A.: The Moderator-Mediator Variable Distinction in Social Psychological Research: Conceptual, Strategic, and Statistical Considerations. Journal of Personality and Social Psychology, 51(6) (1986) 1173-1182

38. Smyth, B., Cotter, P.: MP3 - Mobile Portals, Profiles and Personalization. Web Dynamics (2004) 411-434 